\title{
CHD5 inhibits metastasis of neuroblastoma
}

\author{
Astrid K. Laut ${ }^{1}$, Carmen Dorneburg ${ }^{1}$, Axel Fürstberger ${ }^{2}$, Thomas F. E. Barth ${ }^{3}$, Hans A. Kestler $\mathbb{D}^{2}$, Klaus-Michael Debatin ${ }^{4}$ and \\ Christian Beltinger $\mathbb{D}^{1 凶}$
}

(c) The Author(s) 2021

CHD5, a tumor suppressor at $1 \mathrm{p} 36$, is frequently lost or silenced in poor prognosis neuroblastoma (NB) and many adult cancers. The role of CHD5 in metastasis is unknown. We confirm that low expression of CHD5 is associated with stage 4 NB. Forced expression of CHD5 in NB cell lines with $1 \mathrm{p}$ loss inhibited key aspects of the metastatic cascade in vitro: anchorage-independent growth, migration, and invasion. In vivo, formation of bone marrow and liver metastases developing from intravenously injected NB cells was delayed and decreased by forced CHD5 expression. Genome-wide mRNA sequencing revealed reduction of genes and gene sets associated with metastasis when CHD5 was overexpressed. Known metastasis-suppressing genes preferentially upregulated in CHD5-overexpressing NB cells included PLCL1. In patient NB, low expression of PLCL1 was associated with metastatic disease and poor survival. Knockdown of PLCL1 and of p53 in IMR5 NB cells overexpressing CHD5 reversed CHD5-induced inhibition of invasion and migration in vitro. In summary, CHD5 is a metastasis suppressor in NB.

Oncogene (2022) 41:622-633; https://doi.org/10.1038/s41388-021-02081-0

\section{INTRODUCTION}

Neuroblastoma (NB) is the most common extracranial solid tumor of childhood. Half of the patients harbor metastases and survival of these high-risk patients remains poor [1, 2]. NB metastasizes into lymph nodes, bone marrow, bone, liver, skin and, rarely, lung and brain $[1,2]$.

While the cellular and molecular principles of metastasis, one of the hallmarks of cancer [3], are becoming clearer [4], little is known about the metastatic cascade in NB. Steps in the metastatic sequence of NB include initial dissociation of NB cells from the primary tumor followed by intravasation [5-10], survival of the cells in the circulatory system, and extravasation into organs where the NB cells grow into a metastatic mass $[5,7,11-14]$.

Loss of $1 p$ and $11 q$, gain of $17 q$ and $M Y C N$ amplification are associated with metastatic disease in NB [15]. 1 p36 is frequently deleted in aggressive NB [16-25]. This chromosomal segment contains CHD5 at 1 p36.31 [26-28], amongst several other genes. In $\mathrm{NB}$, as in many adult cancers with $1 \mathrm{p}$ loss, one CHD5 allele is deleted while the second allele is transcriptionally silenced $[29,30]$. Silencing occurs by methylation or other epigenetic mechanism and may involve miRNAs [31]. Deletion and silencing of $\mathrm{CHD} 5$ cause low or absent expression of CHD5 in NB, associated with high-risk factors including advanced stage, and with low survival [29, 32]. CHD5 is a chromodomain-helicase-DNA-binding protein that forms a nucleosome remodeling and deacetylation (NuRD) complex acting on chromatin structure and gene expression [33-35].

CHD5 is a bona fide tumor suppressor gene in NB, as shown by attenuation of clonogenicity in vitro and decrease of tumorigenicity in mice when overexpressed [36]. This tumor suppressor function depends on a functional p19 ${ }^{\mathrm{ARF}}-\mathrm{p} 53$ axis, as determined by chromosome engineering in mice [37].

Since low CHD5 expression is associated with advanced stage, we reasoned that $\mathrm{CHD} 5$ may inhibit metastasis in NB. We now provide experimental evidence that $\mathrm{CHD} 5$ is indeed a metastasis suppressor in NB.

\section{RESULTS}

In patient NB low CHD5 expression is associated with stage 4 metastatic disease and low survival

To confirm and extend previous data of the association of CHD5 mRNA expression with metastasis of NB and patient survival $[29,36]$ we analyzed a large cohort $(n=649)$ of clinically annotated NB. Indeed, low CHD5 mRNA expression is significantly associated with stage 4 , the most metastatic stage (Fig. 1A, left panel) and decreased survival (Fig. 1B). This association is not seen with stage $4 \mathrm{~s}$ (Fig. 1A left panel). In a second, small cohort $(n=34)$ the association of CHD5 with stage 4 was also evident on the protein level (Fig. 1A, right panel).

\section{In NB cell lines with 1p loss CHD5 is a tumor suppressor that may depend on p53}

Next, we assessed CHD5 expression in a panel of 11 NB cell lines. CHD5 mRNA expression was very low, irrespective of MYCN amplification (Fig. 2A, left panel), in line with published data [26]. This corresponded with near-complete absence of CHD5 protein in the cell lines (Fig. 2A, right panel). To assess the effects of CHD5 on cell growth and clonogenicity, we transduced NB cells, which lack CHD5 expression, with either a CHD5 or a mock construct. For this we selected the MYCN-amplified cell lines SK-N-BE(2)C (1p

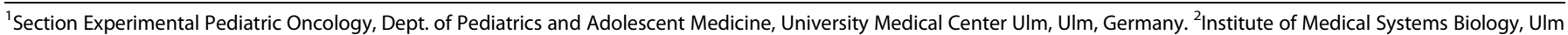

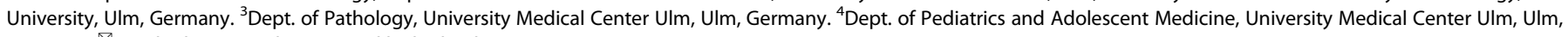
Germany. ${ }^{凶}$ email: Christian.Beltinger@uniklinik-ulm.de
} 
A

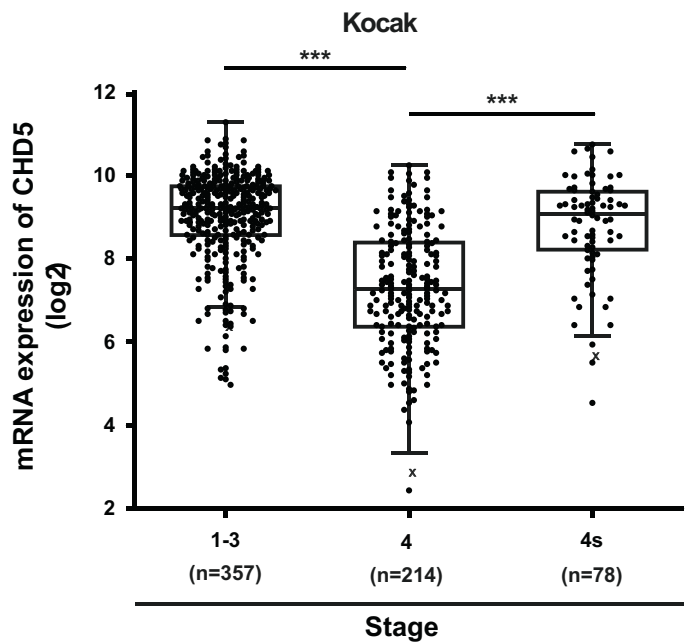

B

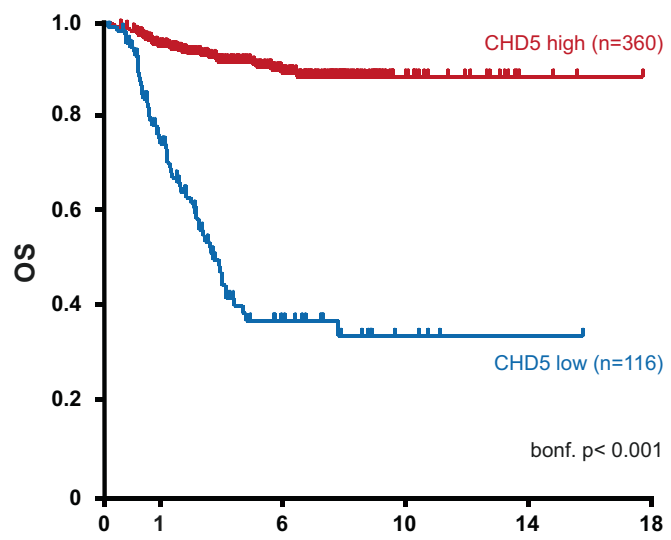

Westermann

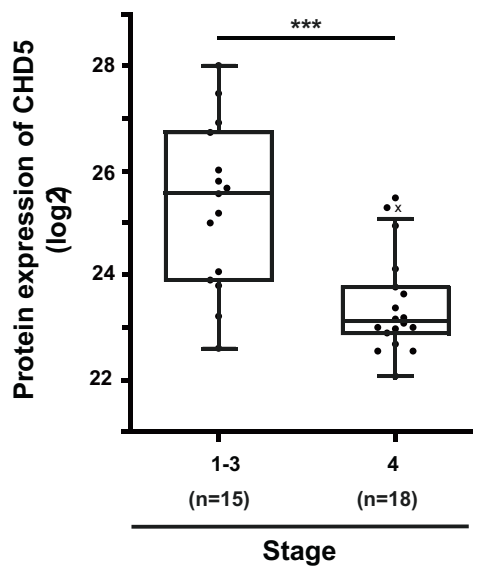

Kocak

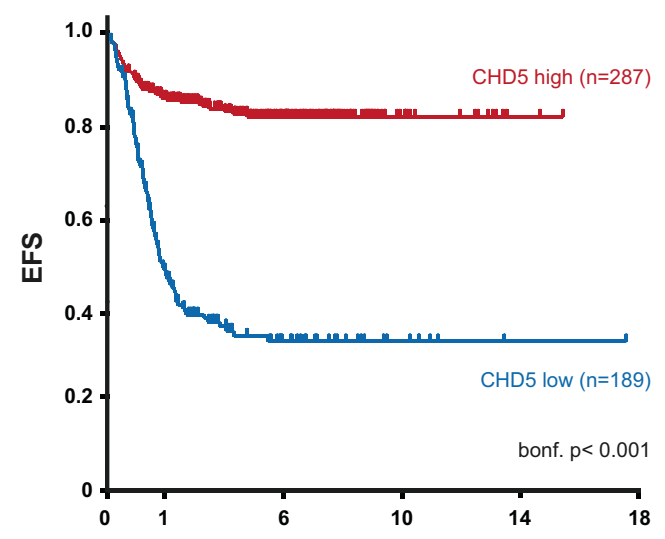

Time from diagnosis $(y)$

Fig. 1 Low expression of CHD5 is associated with aggressive, metastatic disease in patient NB. CHD5 mRNA expression of 649 patient NB (KocakGSE45547) and CHD5 protein expression of 34 patient NB (Westermann-34-LFQ-fw2010prot) were analyzed using the R2 genomics analysis and visualization platform (http://r2.amc.nl). A Low CHD5 expression is associated with stage 4 metastatic disease. CHD5 mRNA expression (left panel) and protein expression (right panel) depending on stage are shown. One-way ANOVA was performed. ${ }^{* * *} p<0.001$. B Low CHD5 mRNA expression is associated with low overall and event-free survival. Kaplan-Meier survival estimates are shown for overall survival (OS) and event-free survival (EFS). Results of the log-rank test are indicated. The cut-off was determined using the scanning method.

loss, p53 non-functional), IMR5 (1p loss, p53 wt) as well as MYCN non-amplified cell lines GI-M-EN (1p loss, p53 wt) and SH-SY5Y (1p wt, p53 wt). CHD5 expression was detected at mRNA and protein levels (Fig. 2B). Forced CHD5 expression trended (without reaching statistical significance) to decrease cell growth of IMR5 and GI-MEN cells, while growth of SK-N-BE(2)C and SH-SY5Y was not affected (Fig. 2C). Clonogenic growth was strongly decreased in IMR5 cells, less pronounced in SK-N-BE(2)C and GI-M-EN cells and unaltered in SH-SY5Y (Fig. 2C). Thus, inhibition of growth and clonogenicity was evident only or more pronounced, respectively, in the 1p deleted, p53 wildtype IMR5 and GI-M-EN cells. In summary, CHD5 is a tumor suppressor in NB cell lines with $1 p$ loss that may depend on functioning p53.

High expression of CHD5 in NB cells with 1p loss decreases proficiency of mechanisms important for metastasis

To address the question whether CHD5 functions as a metastasis suppressor in NB cells, the metastatic cascade was modeled in vitro. First, the ability of the cells to survive without cell-cell and cell-matrix contact (anoikis resistance), required for metastasizing cells in the blood stream, was investigated by challenging the cells to grow in low-density matrigel. IMR5 and SK-N-BE(2)C cells with forced CHD5 expression formed significantly less colonies when deprived of anchorage, while there was no effect of CHD5 overexpression in SH-SY5Y cells (Fig. 3A). GI-MEN cells were unable to grow in soft agar (Fig. 3A). To assess the next major step of the hematogenous metastatic cascade, i.e., adherence of cancer cells to endothelium, adhesion of the NB cells to human umbilical vein endothelial cells (HUVECs) was determined. CHD5 overexpression decreased the capacity of cell lines with $1 \mathrm{p}$ loss to adhere to HUVECs, independent of MYCN status (Fig. 3B). To investigate the subsequent step in the metastatic cascade-invasion into and migration through the matrix of target organs-invasion and migration of the cells in matrigel was assessed. Forced CHD5 expression resulted in reduced capacity of cell lines with $1 p$ loss to invade and migrate in extracellular matrix, while SH-SY5Y cells $(1 \mathrm{p} w \mathrm{wt})$ were unaffected (Fig. 3C). Taken together, high expression of CHD5 in the 1p-deleted SK-N-BE(2)C, IMR5 and GI-M-EN NB cells decreased their metastatic proficiency in vitro. 
A
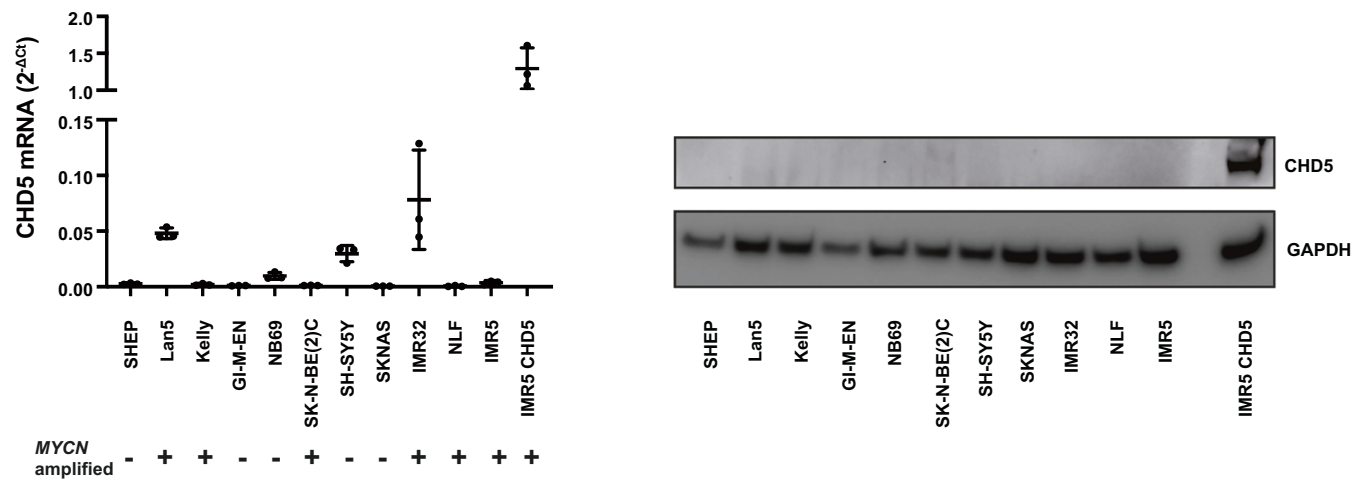

B

SK-N-BE(2)C

IMR5

GI-M-EN

SH-SY5Y

(MYCN amp., 1p loss, p53 mut.) (MYCN amp., 1p loss, p53 wt)

(MYCN non-amp., 1p loss, p53 wt)

(MYCN non-amp., 1p wt, p53 wt)
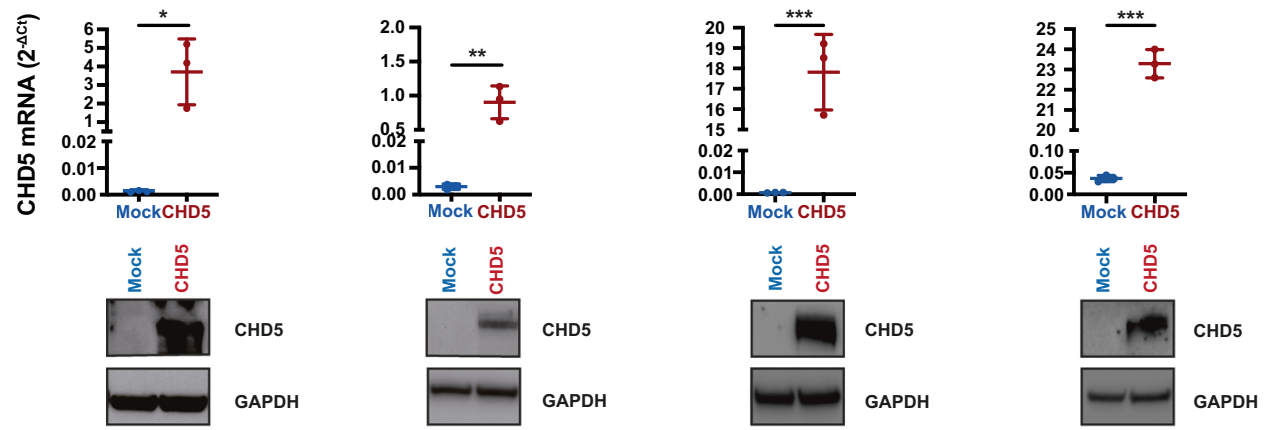

C
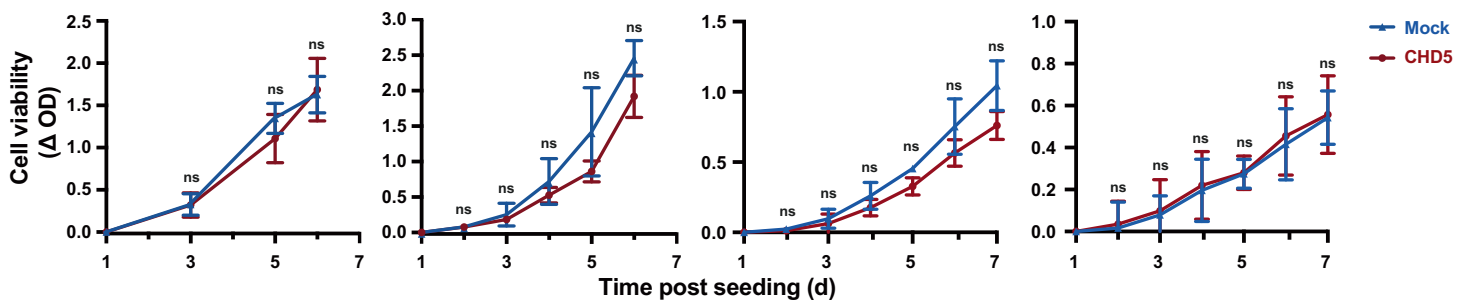

D
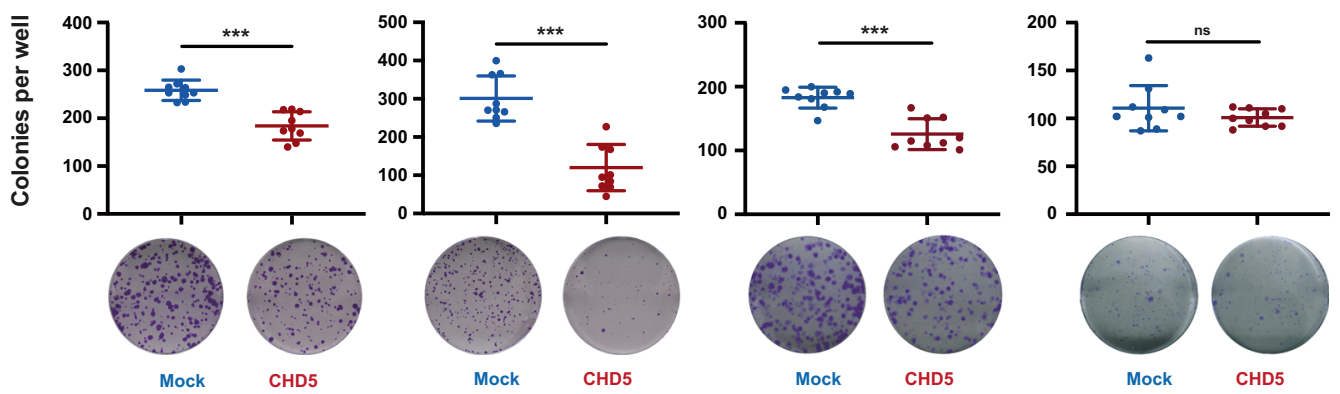

Decreased homing of IMR5 cells with forced CHD5 expression Given that CHD5 inhibited the metastatic cascade in vitro, we investigated its role in experimental metastasis of NB cells in vivo (Fig. 4A). To this end, homing of IMR5 cells was assessed. IMR5 cells with forced CHD5 expression homed significantly less to the bone marrow $2 \mathrm{~h}$ after i.v. injection (Fig. 4B) compared to mock-

transduced cells, and trended (without reaching statistical significance) to home less into liver and lung (Fig. 4C). Interestingly, few cells homed to the lung, in line with the absence of lung metastases in NB patients (Fig. 4C). In summary, forced CHD5 expression reduced homing of IMR5 NB cells to bone marrow and, less so, liver, important metastasis sites in NB patients. 
Fig. 2 CHD5 is a tumor suppressor in NB cell lines with $1 p$ loss. A NB cell lines express very low levels of CHD5. NB cells were subjected to qRTPCR for CHD5 normalized to HPRT expression, MYCN status of the cell lines is indicated (left panel). Cells were investigated by Western Blot analysis for CHD5 protein with GAPDH as loading control (right panel). IMR5 cells with forced expression of CHD5 were used as positive control. B Forced expression of CHD5. SK-N-BE(2)C, IMR5, GI-M-EN and SH-SY5Y cells were stably transduced with either a CHD5 expression or a mock control construct. Cells were analyzed by qRT-PCR and Western Blot analysis for CHD5 expression. Shown is one representative experiment of three independent transductions. C Forced expression of CHD5 reduces cell viability of NB cells with 1p loss. Viable cells growing in 96-well plates were determined by OD measurements after MTT staining. Results are expressed as $\triangle$ OD of day 1. D Forced expression of CHD5 reduces clonogenic growth of NB cells with $1 \mathrm{p}$ loss. For clonogenic growth analysis, cells were stained with crystal violet 10 (SK-N-BE(2)C, IMR5), 11 (GI-M-EN), or 14 (SH-SY5Y) days after plating and colonies per well were counted. Shown is one representative experiment of three independent transductions. Means and SD are shown in (A-D). Statistical analysis was performed using the $t$ test. ns not significant; ${ }^{*} p<0.05 ;{ }^{* *} p<0.01 ;{ }^{* *} p<0.001$. Experiments in (B-D) were repeated at least three times in triplicates, with similar results.

\section{Reduced metastatic burden by forced expression of CHD5}

To investigate whether decreased homing of IMR5 cells with forced CHD5 expression translates into reduced metastatic burden in vivo, metastatic growth in mice was monitored for 4 weeks using bioluminescence in vivo imaging. Forced CHD5 expression decreased tumor incidence and moderately prolonged tumor latency (Fig. 5A). Furthermore, bioluminescent signals were less pronounced in mice injected with cells with forced CHD5 expression, indicating a lower metastatic burden (Fig. 5A upper panel). Mice were sacrificed 4 weeks post injection. Necropsy revealed less macroscopic liver metastases in mice that had received cells with forced CHD5 expression (Fig. 5B). Immunohistochemistry for CD56, a marker for NB cells, confirmed metastases in liver and bone marrow, while no difference in cell morphology or infiltrating growth was detected (Fig. 5C). Immunohistochemistry for CHD5 showed, that the CHD5 overexpression was maintained in the liver metastases (Fig. 5C). Proliferation (Supplementary Fig. 1A) and vascularization (Supplementary Fig. 1B) of the metastases were not altered by forced CHD5 expression. Taken together, these results show that CHD5 decreases the metastatic capacity of NB cells.

\section{GSEA suggests that CHD5 in NB cells is associated with an} antimetastatic status

To gain insights into molecular mechanisms mediating CHD5dependent metastasis inhibition, transcriptome-wide mRNA sequencing followed by GSEA was performed. Upon CHD5 overexpression gene ontologies (GO) related to serine proteinase inhibition, cell-cell adhesion, neuronal differentiation, and inactivation of MAPK-signaling were among the top 10 of 98 significantly enriched gene sets compared to control cells (Fig. 6A). In contrast, gene sets related to glucose metabolism were predominantly enriched in control cells without detectable CHD5 expression compared to CHD5-overexpressing cells (Fig. 6B). These results imply that CHD5 expression in NB cells may be associated with an antimetastatic phenotype, including decreased metabolic flexibility.

\section{PLCL1 appears to contribute to the metastasis-inhibiting} effect of CHD5 in vitro and in situ, and p53 in vitro

To start to delineate potential target genes of $\mathrm{CHD} 5$ that may inhibit metastasis, genes expressed differentially between CHD5overexpressing and control IMR5 cells, as determined by genome-wide mRNA sequencing, were assessed. 52 significantly differentially expressed genes were identified (Fig. 7A and Supplementary Table 1). For further analysis two candidates were selected from this list. First, PLCL1, as this protein is known to be involved in inhibition of tumor growth [38] and metastasis [39]. Second, SERPINB6, as serpin activity was one gene ontology class enriched in GSEA, and because SERPINB6 [40] inhibits metastasis and tumor progression. Given the proposed role of p53 in mediating the tumor-suppressive action of CHD5, p53 was also included in the analysis, although it was not differentially expressed.
As a first step, these genes were analyzed in three clinically annotated NB datasets within the R2 genomics analysis and visualization platform (http://r2.amc.nl) (Fig. 7B and Supplementary Fig. 2A). To assess, whether the candidate genes are independent of MYCN, the analysis was also performed in the MYCN non-amplified subsets of the datasets. SERPINB6 and p53 lose significance when $M Y C N$-amplified tumors are excluded (Fig. $7 B$ and Supplementary Fig. 2A). In contrast, high PLCL1 expression remains significantly associated with nonmetastatic disease independent of MYCN status (Fig. 7B and Supplementary Fig. 2A). In line, high PLCL1 expression is significantly associated with good overall survival in $M Y C N$ non-amplified tumors while SERPINB6 and p53 does not correlate in any of the datasets with patient outcome (Fig. 7C and Supplementary Fig. 2B).

To directly determine the effects of PLCL1, SERPINB6 and p53 on CHD5-mediated inhibition of metastatic mechanisms, IMR5 cells were treated with siRNA against those genes. Knockdown was confirmed at the protein level (Fig. 7D). In CHD5overexpressing cells knockdown of PLCL1 and p53, but not SERPINB6, led to increased capacity to invade and migrate in extracellular matrix (Fig. 7E). In summary, PLCL1 appears to contribute to the metastasis-inhibiting effect of CHD5 in vitro and in patients, and p53 augmented CHD5-associated inhibition of invasion and migration of IMR5 cells in vitro.

\section{DISCUSSION}

CHD5 is frequently lost and silenced in high-risk NB. This work provides evidence that CHD5 inhibits metastasis in this disease. Analysis of patient cohorts confirmed that low CHD5 expression in NB is associated with stage 4 metastatic disease and decreased survival. Low CHD5 expression is not associated with stage $4 \mathrm{~S}$, suggesting that genes other than CHD5 may also control metastasis, and that the impact of $\mathrm{CHD} 5$ on prognosis is not solely due to an effect on metastasis. In vitro experiments validated that CHD5 has a tumor-suppressive function in NB cells. Importantly, CHD5 acts as a metastasis inhibitor in NB cells, independent of its tumor suppressor function. Several lines of evidence in vitro, in vivo and in silico support this notion. Anoikis resistance was decreased in NB cells with high expression of CHD5. Thus, fewer cells would presumably survive in the blood stream during hematogenous dissemination, leading to decreased colonization of target organs. Adherence to the endothelial cells lining the vessels of target organs, and invasion into and migration through the matrix of these organs are key steps in late hematogenous metastasis. Cells expressing CHD5 were less able to perform these tasks in vitro. Along this line, fewer NB cells expressing CHD5 homed to distant organs after i.v. injection into mice. Complementary long-term experiments in mice revealed that CHD5 expression increased latency and decreased incidence of metastases in bone marrow and liver, predilection sites of NB metastases in patients.

Interestingly, forced expression of CHD5 in SH-SY5Y cells, which have not lost $1 p$, did not decrease proliferation, clonogenic 
(MYCN amp., 1p loss, p53 mut.) (MYCN amp., 1p loss, p53 wt)

(MYCN non-amp., 1p loss, p53 wt) (MYCN non-amp., 1p wt, p53 wt)
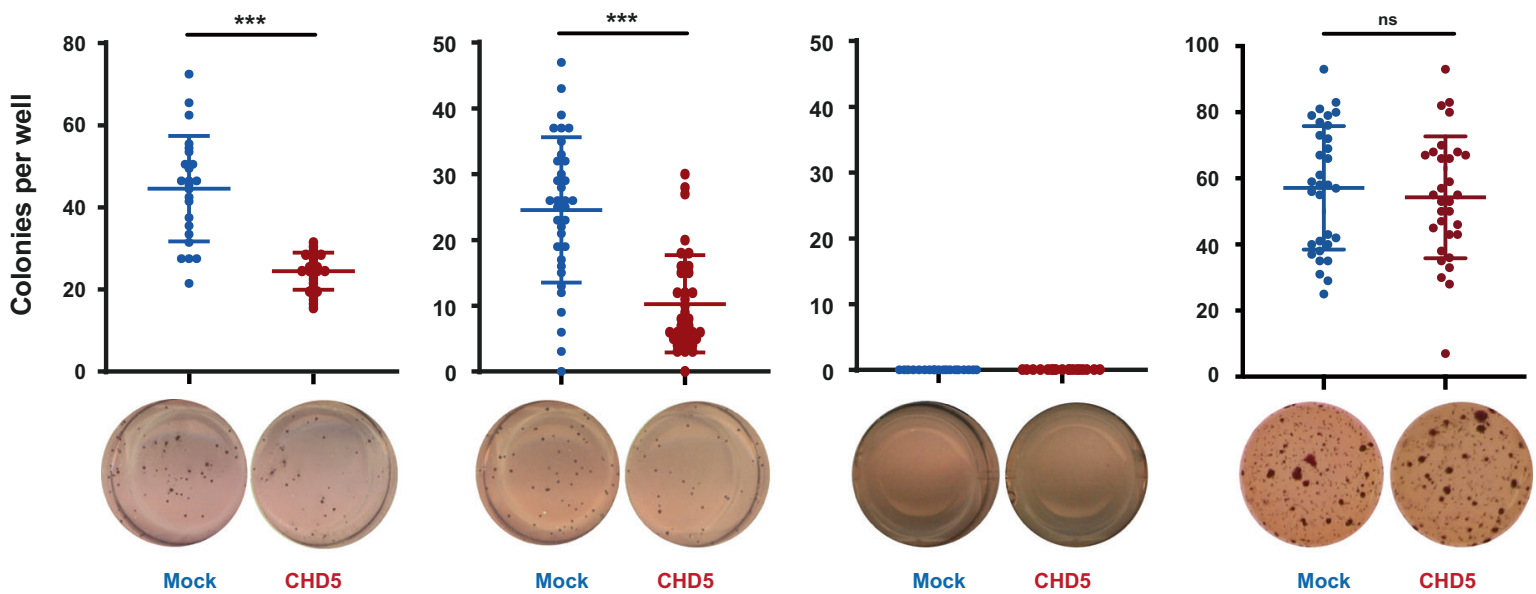

B
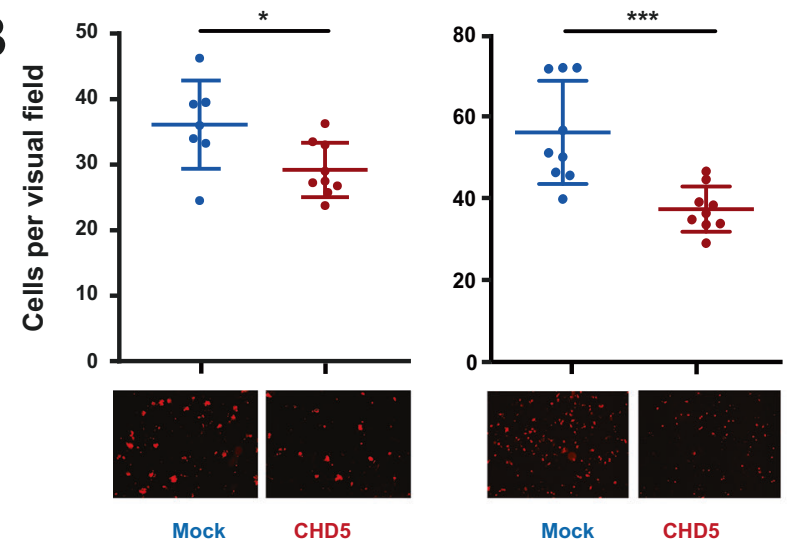

Mock

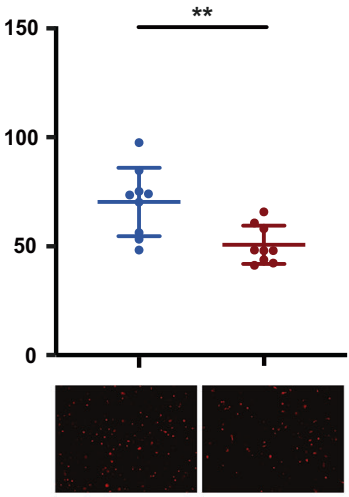

Mock CHD5

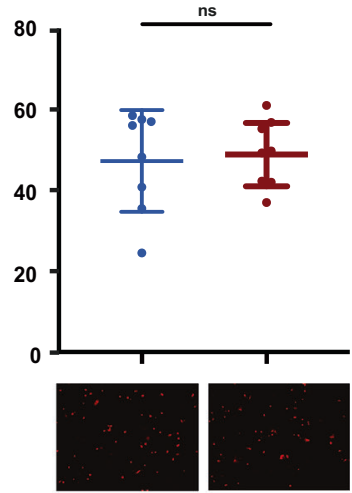

Mock
C

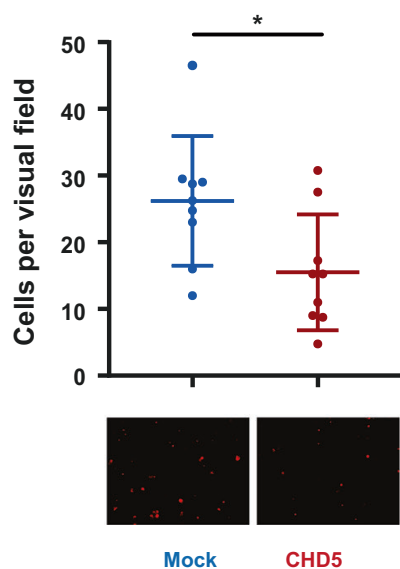

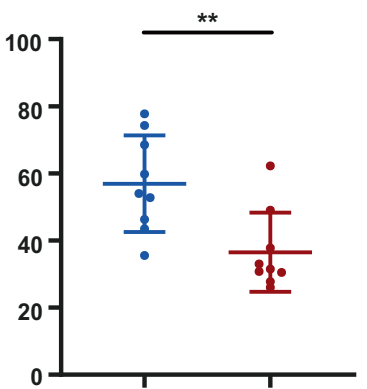

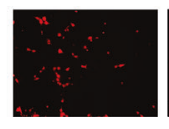

Mock

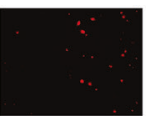

CHD5 growth, anchorage-independent growth, adhesion to endothelial cells, invasion or migration in vitro. This is consistent with the notion that the tumor-suppressive effect of CHD5 in NB is confined to cells with $1 p$ deletion [36].

In mice, the tumor-suppressive effect of $\mathrm{CHD} 5$ proceeds through the p19 ${ }^{A R F}-p 53$ axis [37]. We show in vitro that CHD5

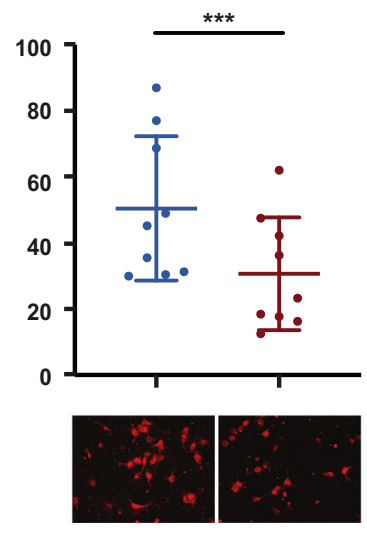

Mock CHD5

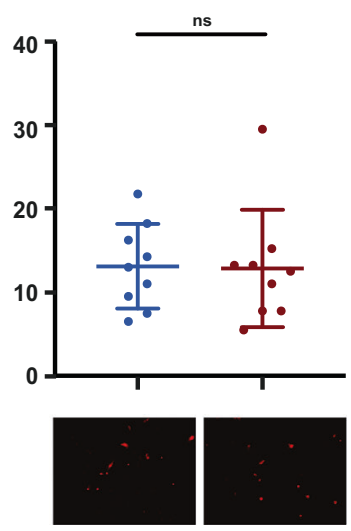

Mock CHD5

does only to some extent decrease the aggressiveness of $\mathrm{p} 53$ mutant SK-N-BE(2)C cells (derived from relapse) or p53-depleted IMR5 cells. This strongly supports the conclusion that p53 is required for the tumor- and metastasis-suppressive effect of CHD5 in NB. As a corollary, these results help to explain the paucity of p53 mutations in NB at time of diagnosis. 
Fig. 3 Forced expression of CHD5 in NB cell lines with 1p loss impairs main steps of the metastatic cascade. A Forced expression of CHD5 decreases anchorage-independent growth. SK-N-BE(2)C, IMR5, GI-M-EN and SH-SY5Y cells transduced with CHD5 expression or mock control constructs were seeded into soft agar in 24-well plates. MTT staining was used to visualize colonies after 12-13 days (IMR5, SK-N-BE(2)C), 8 weeks (SH-SY5Y), or 10 weeks (GI-M-EN). B Forced expression of CHD5 reduces adhesion to HUVECs. Transduced cells were labeled with Dil and seeded on a confluent monolayer of HUVECs. After $1 \mathrm{~h}$, cells were fixed and adhering tumor cells per visual field ( $\times 10$ magnification) were counted. C Forced expression of CHD5 reduces invasion and migration. Transduced cells were labeled with Dil and added to matrigel-coated transwell chambers. The top of the chamber contained medium with $1 \%$ serum and the lower part medium with $10 \%$ serum. $12 \mathrm{~h}$ (IMR5) or $20 \mathrm{~h}$ (SK-N-BE(2)C, GI-M-EN and SH-SY5Y) post seeding, cells that had migrated through the matrigel and membrane, and were attached to the lower surface of the membrane, were fixed. The number of invasive cells per visual field $(\times 20$ magnification $)$ is depicted in the graph. Means and SD are shown in $(\mathbf{A}-\mathbf{C})$. Statistical analysis for $(\mathbf{A}-\mathbf{C})$ was performed using the $t$ test. ns not significant; ${ }^{*} p<0.05 ;{ }^{* *} p<0.01 ;{ }^{* * *} p<$ 0.001 . Experiments were repeated at least three times, with similar results.

Looking beyond p53, none of the known CHD5 target genes provided a satisfactory explanation how CHD5-associated metastasis inhibition may be mediated [34, 41, 42], thus transcriptome analysis was performed. Gene sets associated with glucose metabolism were enriched in NB cells not expressing CHD5, indicating that those cells might cope better with changing and challenging nutrient availability encountered by NB cells during metastasis [43]. Along this line, it is tempting to speculate that CHD5 may inhibit genes involved in the early steps of aerobic glycolysis.

In CHD5-expressing cells gene sets associated with cell-cell adhesion, p53 regulation, inactivation of MAPK signaling and neuronal differentiation were enriched, all consistent with a less metastatic phenotype. The association of CHD5 with neuronal differentiation is intriguing and warrants further investigation, as CHD5 has been described to enhance terminal differentiation of normal neurons [44]. Along this line, undifferentiated sympathetic neuroblasts would be expected to migrate during normal embryogenesis, yet differentiated sympathetic neurons should not, so the effects of CHD5 on the metastatic cascade may partly reflect a secondary effect of neuronal differentiation that is impaired in CHD5-low NB cells.

Searching for specific genes upregulated in CHD5-expressing cells, several lines of evidence suggested SERPINB6 as a promising candidate for mediating CHD5-associated metastasis inhibition in NB. However, when SERPINB6 was knocked down in IMR5 cells with forced expression of CHD5, CHD5-induced inhibition of invasion and migration was not reversed. In addition, MYCN amplification frequently associates with CHD5 loss in NB, so that enhanced SERPINB6 expression could merely be an indirect biomarker of MYCN amplification. Indeed, when correcting for MYCN amplification, SERPINB6 expression lost statistical significance in overall survival. Together, these data suggest that SERPINB6 does not mediate the metastasis-suppressive function of $\mathrm{CHD} 5$ in NB.

Thus, an additional differentially expressed gene potentially mediating CHD5-associated metastasis was explored. Phospholipase $C$ Line 1 (PLCL1) was chosen, as its published functions suggest a role in metastasis. PLCL1 is a catalytically inactive protein binding $\mathrm{PI}(4,5) \mathrm{P} 2$, thus functioning as a decoy for $\mathrm{PI} 3 \mathrm{~K}$, impairing PI3K/AKT signaling and reducing tumor growth [38]. Concomitantly, PLCL1 inhibits polymerization of actin via PI $(3,4,5) P 3-W A V E 2$, thus impairing cell migration [39]. In silico analysis of MYCN non-amplified NB (to assess PLCL1 function independent of $M Y C N$ ) in different independent patient cohorts revealed decreased expression of PLCL1 in stage 4 disease, and decreased patient survival associated with low expression of this gene. Knockdown of PLCL1 in IMR5 cells with forced expression of CHD5 reversed $\mathrm{CHD} 5$-induced inhibition of invasion and migration in vitro. We therefore conclude that PLCL1 may contribute to mediate the metastasis-suppressive function of CHD5.

There are several potential limitations of this study. First, the effects of CHD5 on tumor suppression and metastasis suppression may overlap. However, to disentangle this overlap, isolated steps of the metastatic cascade were assessed in vitro. In addition, NB cells were injected i.v. in the in vivo studies to exclude that different take and growth rates of primary tumors and thus different numbers of metastasizing cells distort the results of the experiments. The obvious limitation of this approach is that the impact of CHD5 on the early steps of metastasis, dissociation of cells from the primary tumor and extravasation, could not be assessed in vivo. Another limitation is the immunodeficient mouse model, which allowed the use of human NB cells but precluded assessment of immunological factors on metastasis. In summary, CHD5 is a metastasis suppressor in NB, mediated in part by PLCL1 and p53. Given, that CHD5 as a chromodomain-helicase modulates expression of many genes, additional, yet to be defined genes may contribute to the metastasis-suppressive effect of CHD5 in NB. Further work to reactivate CHD5 expression or its mediators of metastasis suppression is warranted.

\section{MATERIALS AND METHODS \\ Generation of stable cell lines}

Plasmids pF CAG luc puro (Addgene, Watertown, MA; Cat\# 67501), precision LentiORF RFP control (PerkinElmer, Waltham, MA; Cat\# OHS5832) and precision LentiORF CHD5 (PerkinElmer; Cat\# OHS5898-202623270) were used to generate lentiviral particles in LentiX cells by using Lipofectamine LTX Reagent with PLUS Reagent (Thermo Fisher Scientific, Waltham, MA; Cat\# 15338100) according to the manufacturer's protocol. IMR5 and SK-N-BE(2)C cells were transfected with pF CAG luc puro and stably selected using $0.15-2.75 \mu \mathrm{g} / \mathrm{ml}$ puromycin (Sigma Aldrich, St. Louis, MO). Subsequently, IMR5, SK-N-BE(2)C, SH-SY5Y and GI-M-EN cells were transfected with MOI 5 of either LentiORF RFP control (mock) or LentiORF CHD5 without stop codon (CHD5), and stably selected using $4 \mu \mathrm{g} / \mathrm{ml}(\mathrm{SH}-$ SY5Y and GI-M-EN), $9.75 \mu \mathrm{g} / \mathrm{ml}$ (IMR5), or $13 \mu \mathrm{g} / \mathrm{ml}$ (SK-N-BE(2)C) blasticidin (InvivoGen, San Diego, CA).

All cell lines have been authenticated by short tandem repeat profiles using the GenePrint 10 System (Promega, Mannheim, Germany) according to the manufacturer's protocol. Cells were routinely tested and found to be negative for mycoplasma using the LookOut ${ }^{\circ}$ Mycoplasma PCR Detection Kit (Sigma Aldrich, Cat\# MP0035-1KT) according to the manufacturer's recommendations.

\section{Knockdown of genes by siRNA}

NB cells were transiently transfected with siRNA at $20 \mu \mathrm{M}$ (esiRNA, Sigma Aldrich; Cat\# EHURLUC-20UG, EHU087001-20UG, EHU143431-20UG and EHU123221-20UG) using Lipofectamine RNAiMAX (Invitrogen, Carlsbad, CA) according to the manufacturer's protocol. Downstream experiments were performed $48 \mathrm{~h}$ post transfection.

\section{Quantitative real-time PCR}

qRT-PCR was performed as previously described [45] using the CFX ConnectTM Real-Time system (BioRad, Hercules, CA). For amplification, the following primers were used: CHD5 forward $5^{\prime}$-tgcttaaaggagcccaagtcc- $3^{\prime}$, reverse $5^{\prime}$-tggtcagcgtgtggtaatcc- $3^{\prime}$ and HPRT forward $5^{\prime}$-cctggcgtcgtgattagtga- $3^{\prime}$, reverse $5^{\prime}$-cgagcaagacgttcagtcct- $3^{\prime}$. mRNA expression levels were determined relative to HPRT expression.

\section{Adhesion assay on HUVEC}

HUVEC were grown to monolayers in 24-well plates at standard conditions.

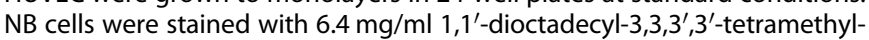


A

I.v. injection of
fluorescent IMR5 cells

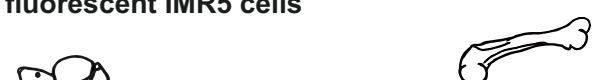

Organ isolation

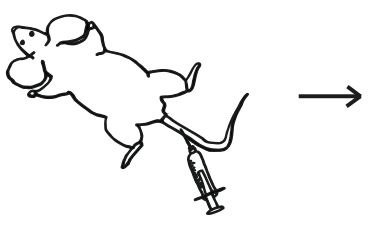

Bone marrow

$\longrightarrow$ FACS analysis

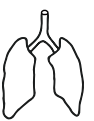

Lung

Liver

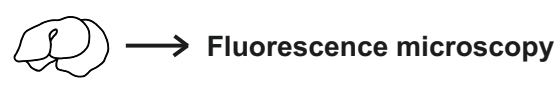

B

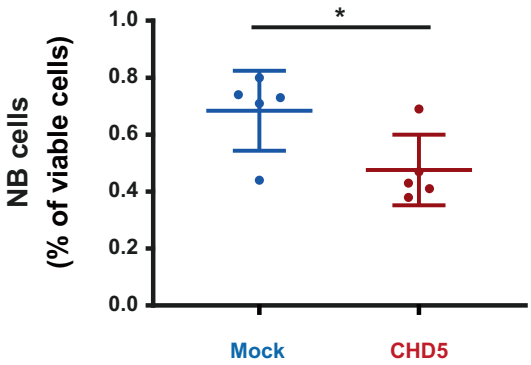

Bone marrow

C
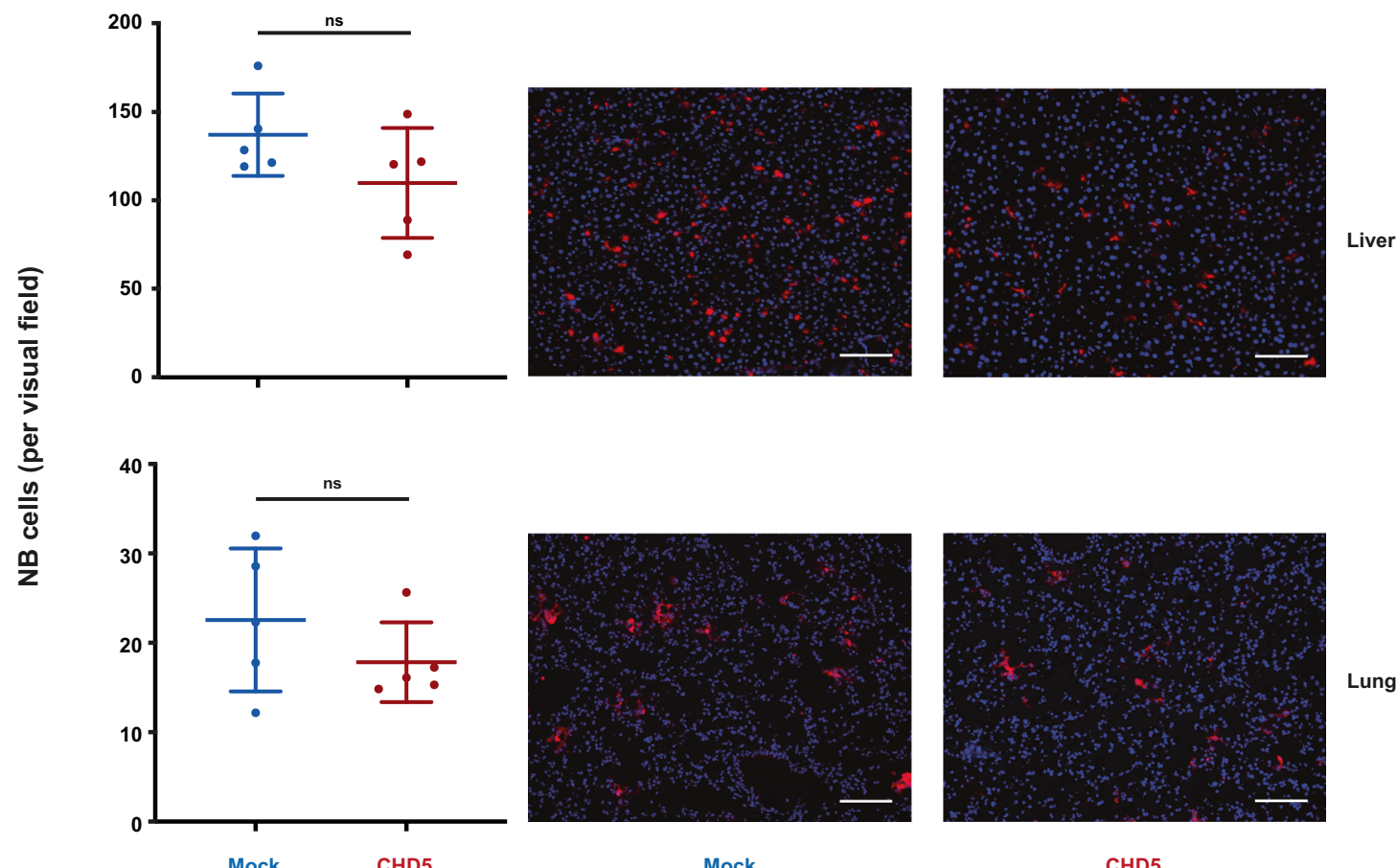

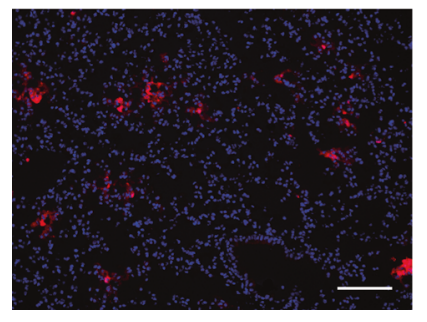

Mock

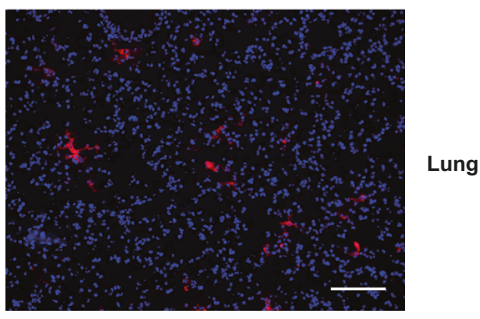

CHD5

Fig. 4 Forced expression of CHD5 impairs homing of intravenously injected IMR5 cells. A Schematic of the in vivo experiment to analyze homing of NB cells in mice. $5 \times 10^{6}$ Dil-labeled IMR5 cells were injected into the tail vein of RAG2 ${ }^{-1-} \mathrm{C \gamma c}^{-1-}$ mice (five animals per group). $2 \mathrm{~h}$ after injection, mice were sacrificed. Bone marrow was subjected to FACS analysis, and liver and lung to fluorescence microscopy to determine the number of homed IMR5 cells. B Decreased homing of IMR5 with forced expression of CHD5 to bone marrow. Bone marrow of both femurs was extracted and the number of Dil-positive cells was determined using FACS analysis. C Decreased homing of IMR5 with forced expression of CHD5 to liver and lung. Lung and liver tissue were formalin-fixed, cryo-embedded, and subsequently counterstained with DAPI. The number of Dil-positive cells per visual field (30 fields per organ and mouse) is indicated. Scale bar equals $100 \mu \mathrm{m}$. Means and SD are shown for both panels. The $t$ test was used for statistical analysis. ns not significant; ${ }^{*} p<0.05$. 
A
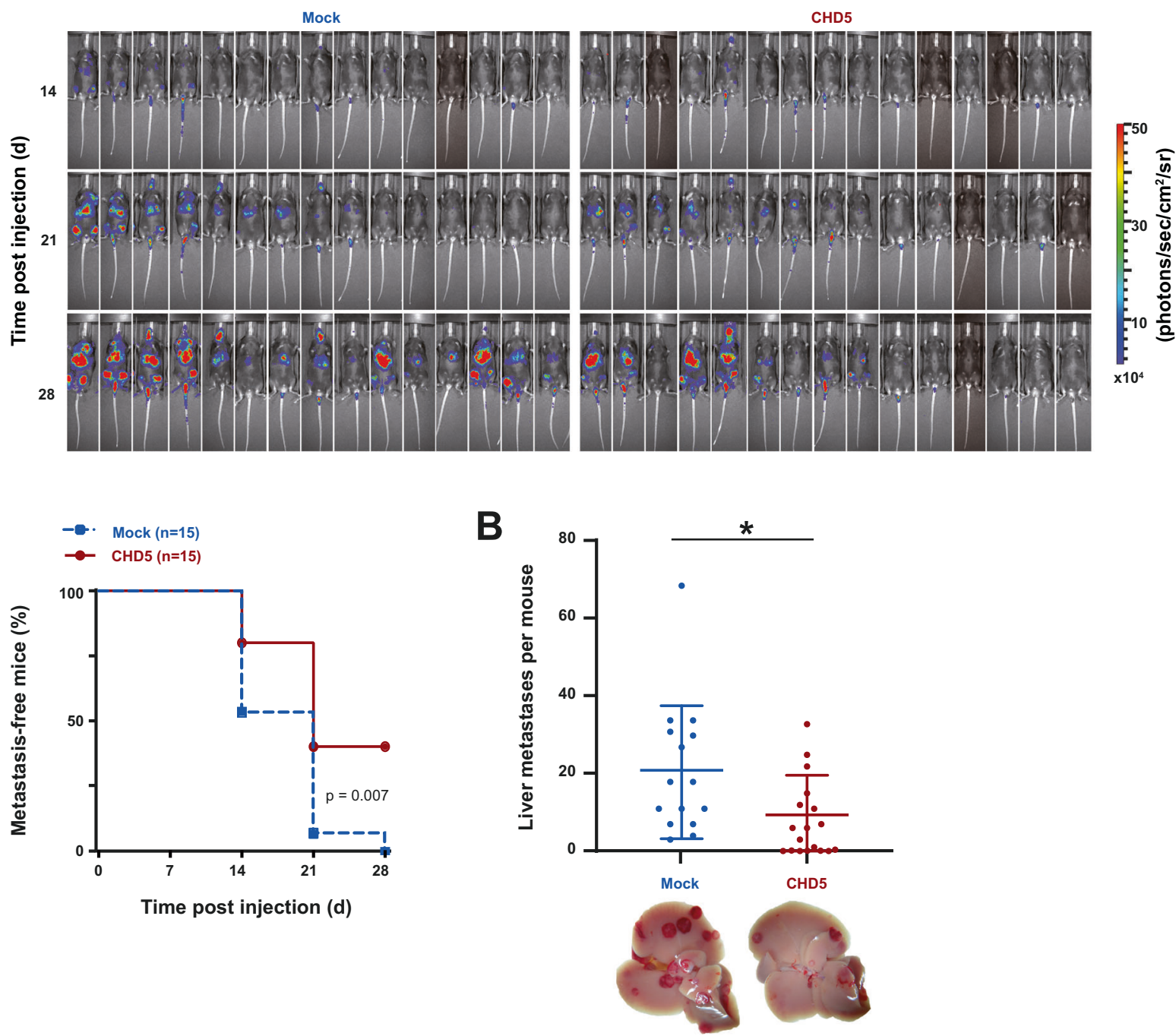

C

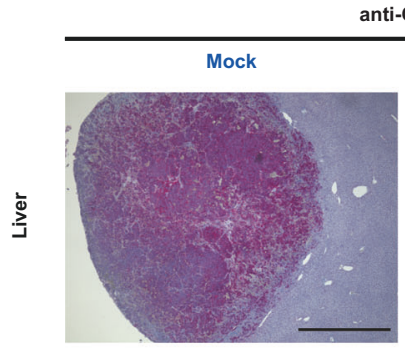

anti-CD56
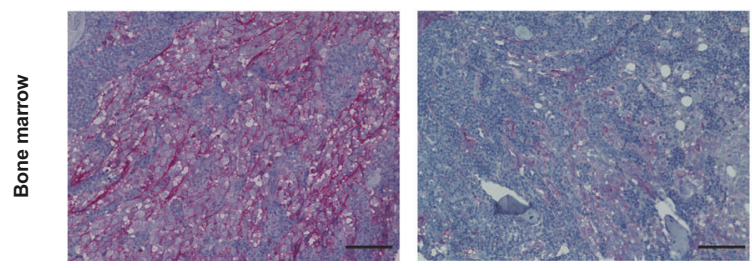

anti-CHD5

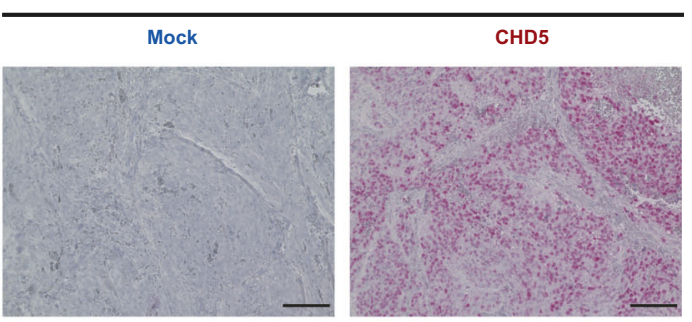

indocarbocyanine perchlorate (Dil, Thermo Fisher Scientific) and $5 \times 10^{4}$ cells $/ \mathrm{ml}$ were seeded per well on top of the HUVEC monolayer. After $1 \mathrm{~h}$ incubation at standard conditions, cells were washed twice before fixing with $3.7 \%$ paraformaldehyde. Dil-labeled cells in 12 visual fields were counted using a Keyence BZ-9000 microscope.

\section{Transwell invasion assay}

Single cells were stained with $10 \mathrm{mg} / \mathrm{ml}$ Dil and $5 \times 10^{4}$ (IMR5, GI-M-EN, SHSY5Y) or $2.5 \times 10^{4}$ (SK-N-BE(2)C) cells were seeded in medium with $1 \%$ FBS on top of matrigel-coated PET membranes with $8 \mu \mathrm{m}$ pores (Corning, Corning, NY) placed in companion plates (Corning). The lower chamber 
Fig. 5 Forced expression of CHD5 inhibits formation and growth of metastases. A Forced expression of CHD5 increases latency and decreases incidence of metastases. $1 \times 10^{6}$ transduced IMR5 cells stably expressing the firefly luciferase gene were injected into the tail vein of $\mathrm{RAG2}^{-1-} \mathrm{C \gamma c}^{-/-}$mice. Development of metastases was assessed once per week for 3 weeks by luminescence imaging following luciferin injection. The absence of metastases is depicted in the Kaplan-Meier plot. The log-rank test was used for statistical analysis. B Forced expression of CHD5 reduces the number of metastases. Mice were sacrificed $30 \mathrm{~d}$ post-tumor cell injection and organs were formalin-fixed. The number of metastases visible on the liver surface was determined. Means and SD are shown for each group. Statistical analysis was performed using the $t$ test. ${ }^{*} p<0.05$. C Immunohistochemistry confirms metastases and their persistent CHD5 overexpression. Formalin-fixed, paraffin-embedded liver and bone marrow samples from day 28 were subjected to immunohistochemistry for CD65, a neuroblastoma marker, and CHD5. Representative pictures for each group are shown. Scale bars equal $1 \mathrm{~mm}$ (liver) or $100 \mu \mathrm{m}$ (bone marrow).

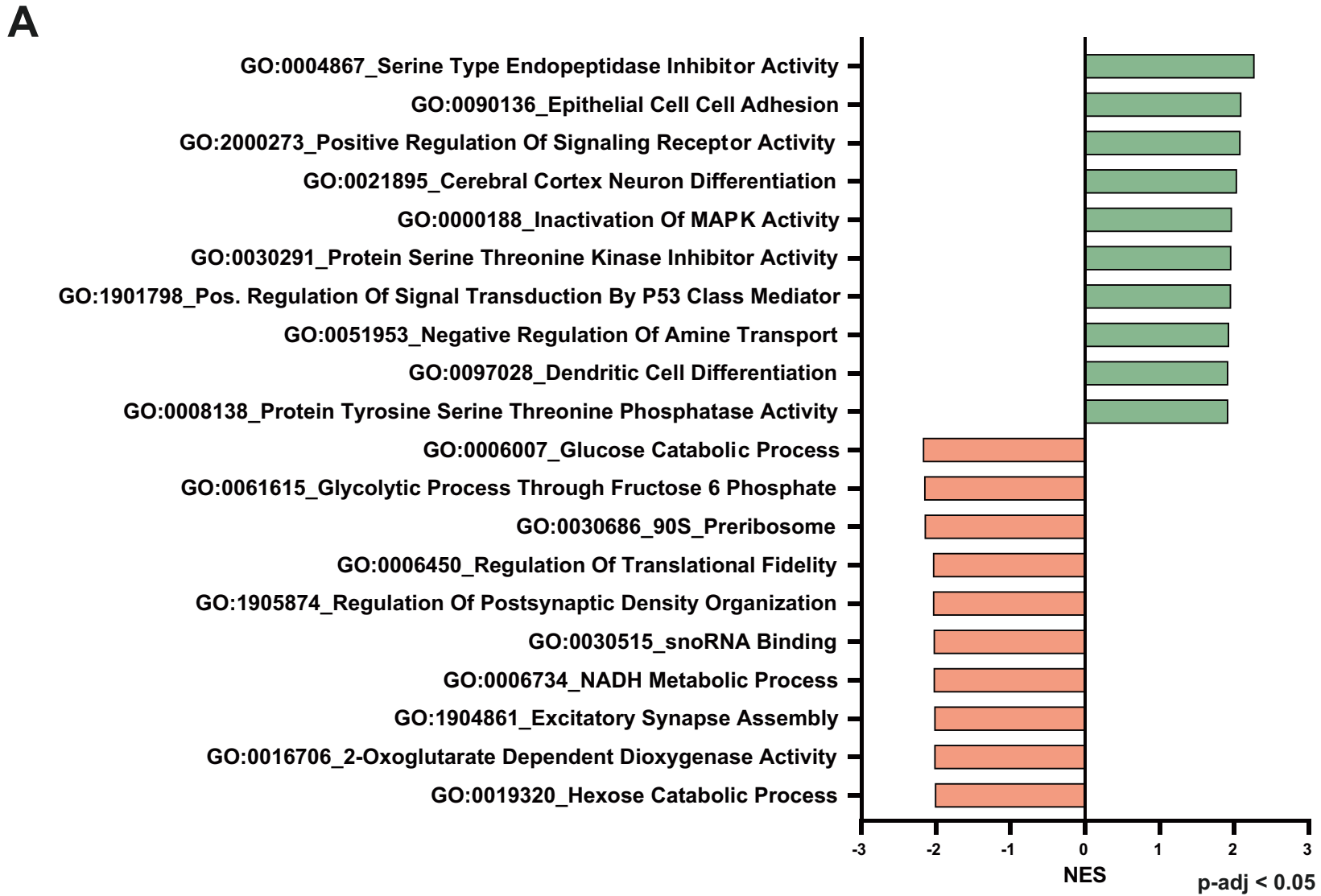

B

GO_Epithelial Cell Cell Adhesion

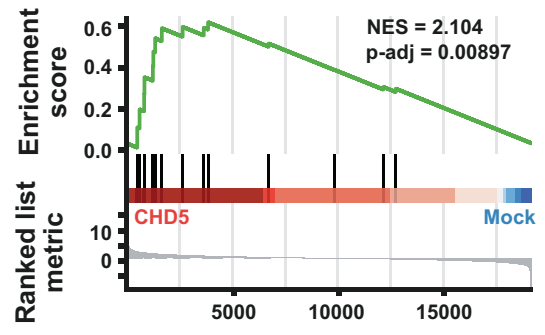

GO_Glucose Catabolic Process

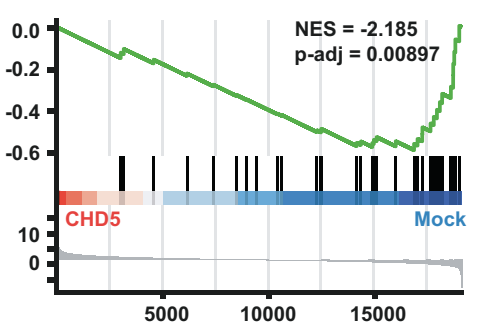

GO_Glycolytic Process Through Fructose 6 Phosphate

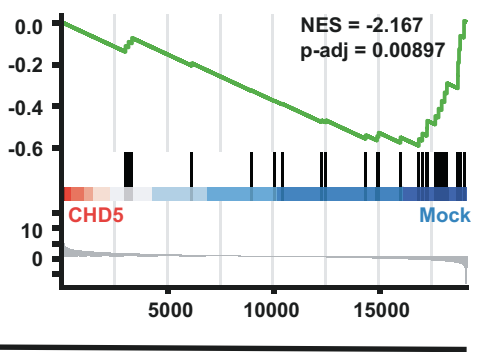

Rank in Ordered Dataset

Fig. 6 Gene sets indicative of an antimetastatic status are enriched in CHD5-overexpressing NB cells. A Enrichment and depletion of GO classes associated with antimetastatic mechanisms and metabolic flexibility, respectively. The transcriptomes of three independent sets of IMR5 cells overexpressing CHD5 and corresponding control cells were determined by mRNA sequencing. Gene set enrichment analysis (GSEA) was performed using the gene ontology (GO) classes. Results were filtered for p-adjusted and ranked according to the normalized enrichment score (NES) to identify the most relevant GO classes. The table shows the top ten significantly $(p<0.001)$ enriched (green) and depleted (red) GO classes. B Increased cell-cell adhesion and decreased glucose metabolism by CHD5 expression. GSEA plots are shown. Based on the ranked list metric, enrichment scores (green curves) and position of genes (black vertical lines) are shown. NES and p-adjusted values are shown. 
A

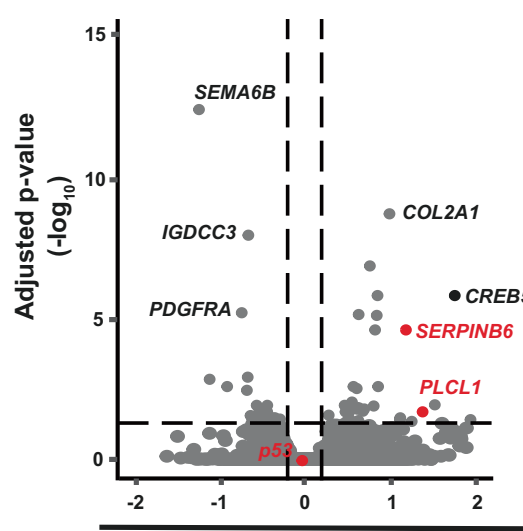

Adj. p-value $<0.05$

Log2fold change $<-0.2$ and $>0.2$

Total $=60624$ transcript variants

Kocak

B

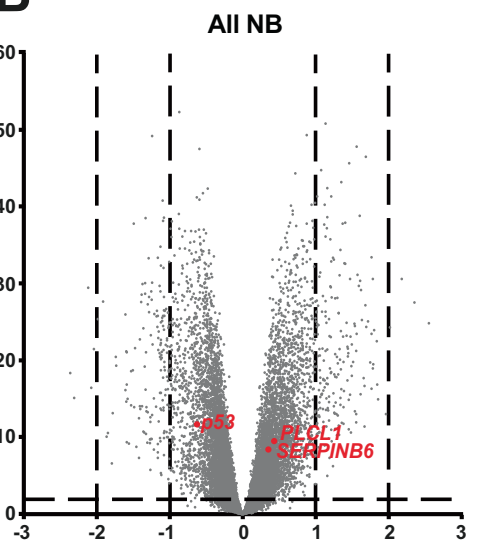

MYCN non-amp. NB

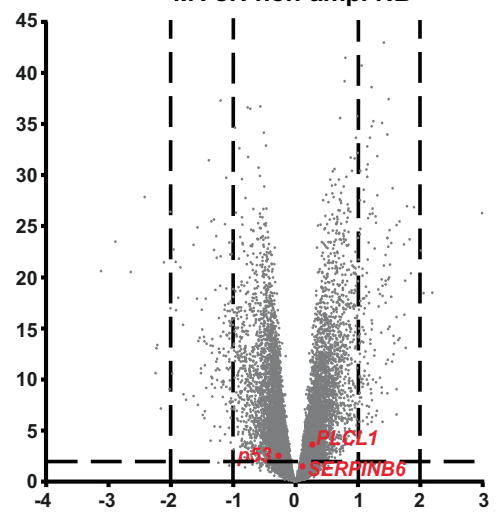

Log2 fold change

C

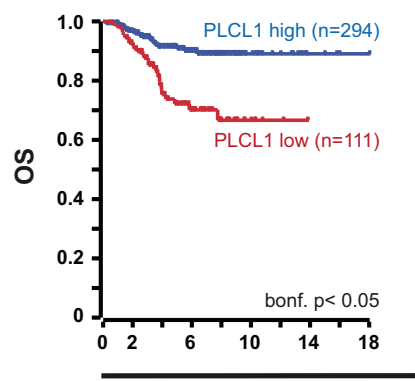

Kocak (MYCN non-amp. NB)
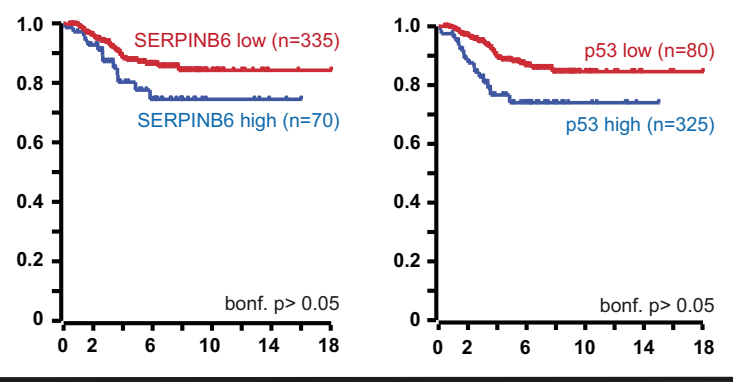

Time from diagnosis $(y)$

D

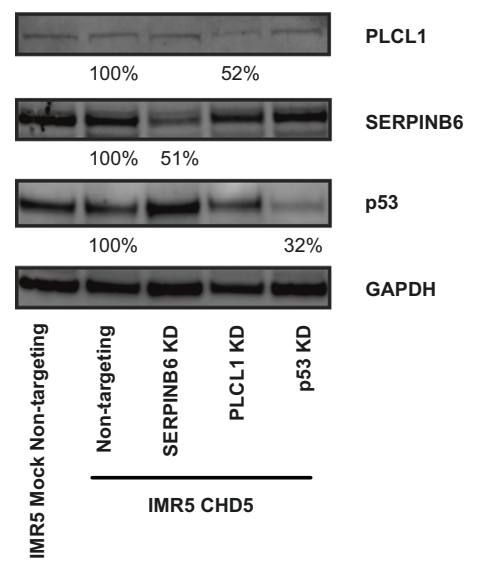

$\mathbf{E}$

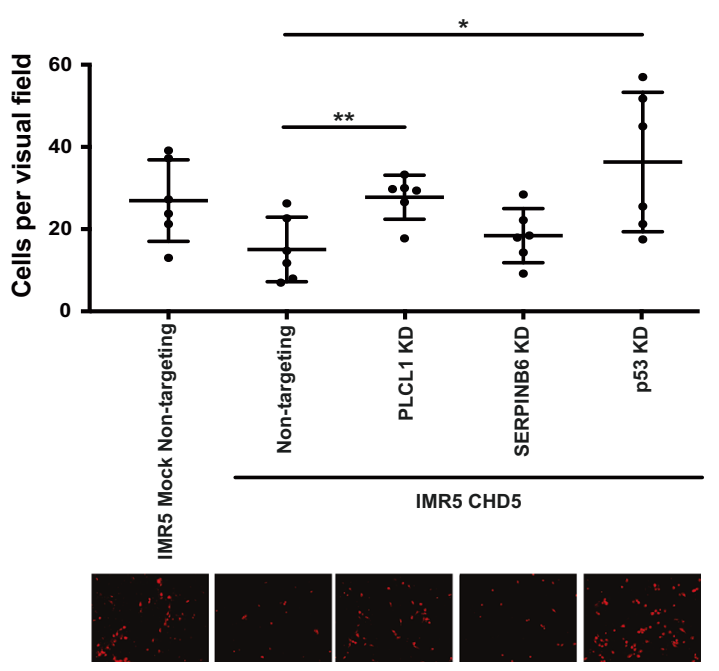

was filled with medium containing $10 \%$ FCS. Cells were fixed $12 \mathrm{~h}$ (IMR5) or $20 \mathrm{~h}$ (SK-N-BE(2)C, GI-M-EN, SH-SY5Y) post seeding with 3.7\% paraformaldehyde. Membranes were cut out and embedded. Cells, which had migrated through the membrane, were analyzed in 12 visual fields using a Keyence BZ-9000 micoscope.

\section{Animal experiments}

To analyze initial homing, $5 \times 10^{6}$ IMR5 cells were stained with $10 \mathrm{mg} / \mathrm{ml}$ Dil, suspended in $200 \mu \mathrm{l} \mathrm{PBS}$ and injected in the tail veins of 6-8 weeks old RAG2 ${ }^{-1-} \mathrm{Cyc}^{-1-}$ mice ( $n=5$ per group). Mice were sacrificed $2 \mathrm{~h}$ post injection and organs were collected for cryosectioning. 30 slides per organ 
Fig. 7 PLCL1 contributes to the metastasis-inhibiting effect of CHD5 in vitro and in situ, and p53 in vitro. A PLCL1 and SERPINB6 are among the top differentially expressed genes. The mRNA sequencing-derived transcriptomes of IMR5 cells overexpressing CHD5 and corresponding control cells were analyzed for differentially expressed genes. Gray and red dots in the volcano plot represent genes differentially expressed between both groups. B High PLCL1 expression in patient NB is associated with nonmetastatic disease independent of $M Y C N$ amplification. Gene expression depending on stage is shown in a clinically annotated NB dataset (Kocak, GSE45547, $n=571 ;$ R2 genomics analysis and visualization platform) for all NB of the dataset (left panel) or for MYCN non-amplified NB (right panel). Gray and red dots in the volcano plot represent genes differentially expressed between stage 1-3 versus stage 4 patients. C High PLCL1 expression is associated with increased overall survival. Kaplan-Meier overall survival (OS) estimates for expression of PLCL1, SERPINB6 and p53 in MYCN non-amplified NB of the Kocak dataset are shown. Results of the log-rank test are indicated. The cut-off was determined using the scanning method. D Knockdown of p53, PLCL1 or SERPINB6. IMR5 cells stably transduced with either CHD5 expression or a mock control construct were treated for $48 \mathrm{~h}$ with siRNA against p53, PLCL1 or SERPINB6. Cells were analyzed by Western Blot for PLCL1, SERPINB6, and p53 expression. Shown is one representative experiment of three independent siRNA transductions. E Downregulation of PLCL1 and p53 but not SERPINB6 in CHD5overexpressing cells enhances invasion and migration. Transduced and siRNA-treated cells were labeled with Dil and added to matrigelcoated transwell chambers. The top of the chamber contained medium with $1 \%$ serum and the lower part medium with $10 \%$ serum. $12 \mathrm{~h}$ post seeding, cells that had migrated through the matrigel and membrane, and were attached to the lower surface of the membrane, were fixed. The number of invasive cells per visual field ( $\times 20$ magnification) is depicted in the graph. Means and SD are shown. Statistical analysis was performed using the $t$ test. ${ }^{*} p<0.05,(n=3$ in duplicates).

were prepared and stained with Hoechst 33342 (1:1000; Thermo Fisher Scientific). Bone marrow samples were analyzed by flow cytometry using an Attune NxT cytometer (Thermo Fisher Scientific) and FlowJo 10.5.3 software (RRID:SCR_008520, Tree Star, Olten, Switzerland).

For analysis of metastases formation, $1 \times 10^{6}$ cells were injected intravenously in 6-8 weeks old $\mathrm{RAG2}^{-1-} \mathrm{cyc}^{-1-}$ mice $(n=15$ per group, female and male mice were equally assigned to both groups). Mice were analyzed by bioluminescence imaging once per week (Xenogen, IVIS 200, Caliper Life Sciences, Mainz, Germany). Four weeks post injection, mice were sacrificed and tissues were collected for histological staining. All experiments were performed according to institutional and state guidelines for the care and protection of animals.

\section{mRNA sequencing and bioinformatics analyses}

Total RNA was isolated as described in Supplementary Materials and methods. Random-primed cDNA library preparation and stranded mRNASeq (NextSeq 500, $1 \times 75$ bp, 30 million reads, Illumina, San Diego, CA) of three samples per condition were performed by the EMBL Genomics Core Facility (Heidelberg, Germany). Reads were mapped to the human hg38 reference genome using bowtie2 (version 2.3.5.1, RRID:SCR_016368), assembled into putative transcripts and annotated. Average alignment rate was $87.2 \%$ (86.99-87.51\%). Aligned files were sorted using samtools sort (version 1.9, RRID:SCR_002105) and read counting was performed using htseq-count from htseq (version 0.9.1). Differential expression analysis was performed using the R/Bioconductor DESeq2 (version 1.28.1, RRID:SCR_015687) package. Gene Set Enrichment Analysis (GSEA) was conducted by mapping results to the GO database (Molecular Signature Database (MSigDB); R-package msigdbr version 7.2.1) using the R/fGSEA (version 1.14.0) package. Data can be accessed via GSE183824.

\section{Statistical analysis}

Data were analyzed using GraphPad Prism 8.4.3 software (RRID: SCR_002798, GraphPad Software, San Diego, CA) and are presented as the mean $\pm S D$. For in vitro analyses no prespecified effect size was required. Three independent experiments were performed, unless stated otherwise. Appropriate sample size for animal experiments was determined by biometric evaluation. The unpaired, two-sided Student's $t$ test was used for comparison of two groups. One-way ANOVA was used for multiple group comparisons. Variance between groups was checked and found to be sufficiently similar. The log-rank (Mantel-Cox) test was used for survival studies. Results were considered significant if $p<0.05$.

Additional information can be found in Supplementary Materials and methods.

\section{REFERENCES}

1. Maris JM. Recent advances in neuroblastoma. N Engl J Med. 2010;362:2202-11.

2. Matthay KK, Maris JM, Schleiermacher G, Nakagawara A, Mackall CL, Diller L, et al. Neuroblastoma Nat Rev Dis Prim. 2016;2:16078.

3. Hanahan D, Weinberg RA. Hallmarks of cancer: the next generation. Cell. 2011;144:646-74.

4. Lambert AW, Pattabiraman DR, Weinberg RA. Emerging biological principles of metastasis. Cell. 2017;168:670-91.
5. Byrne FL, Yang L, Phillips PA, Hansford LM, Fletcher Jl, Ormandy CJ, et al. RNAimediated stathmin suppression reduces lung metastasis in an orthotopic neuroblastoma mouse model. Oncogene. 2014;33:882-90.

6. Delloye-Bourgeois C, Bertin L, Thoinet K, Jarrosson L, Kindbeiter K, Buffet T, et al. Microenvironment-driven shift of cohesion/detachment balance within tumors induces a switch toward metastasis in neuroblastoma. Cancer Cell. 2017;32:427-43.e8.

7. Fife CM, Sagnella SM, Teo WS, Po'uha ST, Byrne FL, Yeap YYC, et al. Stathmin mediates neuroblastoma metastasis in a tubulin-independent manner via RhoA/ ROCK signaling and enhanced transendothelial migration. Oncogene. 2017;36:501-11.

8. Zhu S, Zhang X, Weichert-Leahey N, Dong Z, Zhang C, Lopez G, et al. LMO1 synergizes with MYCN to promote neuroblastoma initiation and metastasis. Cancer Cell. 2017;32:310-23.e5.

9. Wu P-Y, Yu I-S, Lin Y-C, Chang Y-T, Chen C-C, Lin K-H, et al. Activation of aryl hydrocarbon receptor by kynurenine impairs progression and metastasis of neuroblastoma. Cancer Res. 2019;79:5550-62.

10. Chen D, Cox J, Annam J, Weingart M, Essien G, Rathi KS, et al. LIN28B promotes neuroblastoma metastasis and regulates PDZ binding kinase. Neoplasia. 2020;22:231-41.

11. Xiang X, Zhao X, Qu H, Li D, Yang D, Pu J, et al. Hepatocyte nuclear factor 4 alpha promotes the invasion, metastasis and angiogenesis of neuroblastoma cells via targeting matrix metalloproteinase 14. Cancer Lett. 2015;359:187-97.

12. Fabian J, Opitz D, Althoff K, Lodrini M, Hero B, Volland R, et al. MYCN and HDAC5 transcriptionally repress CD9 to trigger invasion and metastasis in neuroblastoma. Oncotarget. 2016;7:66344-59.

13. Seong BKA, Fathers KE, Hallett R, Yung CK, Stein LD, Mouaaz S, et al. A metastatic mouse model identifies genes that regulate neuroblastoma metastasis. Cancer Res. 2017;77:696-706.

14. Bhoopathi P, Pradhan AK, Bacolod MD, Emdad L, Sarkar D, Das SK, et al. Regulation of neuroblastoma migration, invasion, and in vivo metastasis by genetic and pharmacological manipulation of MDA-9/Syntenin. Oncogene. 2019;38:6781-93.

15. Ara T, DeClerck YA. Mechanisms of invasion and metastasis in human neuroblastoma. Cancer Metastasis Rev. 2007;25:645-57.

16. Cheng NC, Van Roy N, Chan A, Beitsma M, Westerveld A, Speleman F, et al. Deletion mapping in neuroblastoma cell lines suggests two distinct tumor suppressor genes in the 1p35-36 region, only one of which is associated with N-myc amplification. Oncogene. 1995;10:291-7.

17. Caron H, Peter M, van Sluis P, Speleman F, de Kraker J, Laureys G, et al. Evidence for two tumour suppressor loci on chromosomal bands 1p35-36 involved in neuroblastoma: one probably imprinted, another associated with $\mathrm{N}$-myc amplification. Hum Mol Genet. 1995;4:535-9.

18. White PS, Maris JM, Beltinger C, Sulman E, Marshall HN, Fujimori M, et al. A region of consistent deletion in neuroblastoma maps within human chromosome 1p36.2-36.3. Proc Natl Acad Sci. 1995;92:5520-4.

19. Gehring M, Berthold F, Edler L, Schwab M, Amler LC. The 1p deletion is not a reliable marker for the prognosis of patients with neuroblastoma. Cancer Res. 1995;55:5366.

20. Martinsson T, Sjöberg RM, Hedborg F, Kogner P. Deletion of chromosome 1p loci and microsatellite instability in neuroblastomas analyzed with short-tandem repeat polymorphisms. Cancer Res. 1995;55:5681-6.

21. White $P$, Maris J, Sulman E, Jensen S, Kyemba S, Beltinger $C$, et al. Molecular analysis of the region of distal $1 \mathrm{p}$ commonly deleted in neuroblastoma. Eur $\mathrm{J}$ Cancer. 1997;33:1957-61. 
22. Martinsson T, Sjöberg R, Hallstensson $K$, Nordling $M$, Hedborg F, Kogner $P$. Delimitation of a critical tumour suppressor region at distal $1 \mathrm{p}$ in neuroblastoma tumours. Eur J Cancer. 1997;33:1997-2001.

23. White PS, Thompson PM, Seifried BA, Sulman EP, Jensen SJ, Guo C, et al. Detailed molecular analysis of 1p36 in neuroblastoma. Med Pediatr Oncol. 2001;36:37-41.

24. Caron $\mathrm{H}$, Spieker N, Godfried $M$, Veenstra $M$, van Sluis $P$, de Kraker J, et al. Chromosome bands 1 p35-36 contain two distinct neuroblastoma tumor suppressor loci, one of which is imprinted. Genes Chromosomes Cancer. 2001;30:168-74.

25. Bauer A, Savelyeva L, Claas A, Praml C, Berthold F, Schwab M. Smallest region of overlapping deletion in $1 \mathrm{p} 36$ in human neuroblastoma: a $1 \mathrm{Mbp}$ cosmid and PAC contig. Genes Chromosomes Cancer. 2001;31:228-39.

26. Thompson PM, Gotoh T, Kok M, White PS, Brodeur GM. CHD5, a new member of the chromodomain gene family, is preferentially expressed in the nervous system. Oncogene. 2003;22:1002-11.

27. White PS, Thompson PM, Gotoh T, Okawa ER, Igarashi J, Kok M, et al. Definition and characterization of a region of $1 \mathrm{p} 36.3$ consistently deleted in neuroblastoma. Oncogene. 2005;24:2684-94.

28. Okawa ER, Gotoh T, Manne J, Igarashi J, Fujita T, Silverman KA, et al. Expression and sequence analysis of candidates for the $1 \mathrm{p} 36.31$ tumor suppressor gene deleted in neuroblastomas. Oncogene. 2008;27:803-10.

29. Koyama H, Zhuang T, Light JE, Kolla V, Higashi M, McGrady PW, et al. Mechanisms of CHD5 Inactivation in Neuroblastomas. Clin Cancer Res. 2012;18:1588-97.

30. Kolla V, Zhuang T, Higashi M, Naraparaju K, Brodeur GM. Role of CHD5 in human cancers: 10 years later. Cancer Res. 2014;74:652-8.

31. Naraparaju K, Kolla V, Zhuang T, Higashi M, lyer R, Kolla S, et al. Role of microRNAs in epigenetic silencing of the CHD5 tumor suppressor gene in neuroblastomas. Oncotarget. 2016;7:15977-85.

32. Garcia I, Mayol G, Rodríguez E, Suñol M, Gershon TR, Ríos J, et al. Expression of the neuron-specific protein CHD5 is an independent marker of outcome in neuroblastoma. Mol Cancer. 2010;9:277.

33. Quan J, Yusufzai T. The tumor suppressor chromodomain helicase DNA-binding protein 5 (CHD5) remodels nucleosomes by unwrapping. J Biol Chem. 2014;289:20717-26.

34. Paul S, Kuo A, Schalch T, Vogel H, Joshua-Tor L, McCombie WR, et al. Chd5 requires PHD-mediated histone 3 binding for tumor suppression. Cell Rep. 2013;3:92-102.

35. Kolla V, Naraparaju K, Zhuang T, Higashi M, Kolla S, Blobel GA, et al. The tumour suppressor CHD5 forms a NuRD-type chromatin remodelling complex. Biochem J. 2015;468:345-52.

36. Fujita T, Igarashi J, Okawa ER, Gotoh T, Manne J, Kolla V, et al. CHD5, a Tumor Suppressor Gene Deleted From 1p36.31 in Neuroblastomas. JNCI Journal of the National Cancer Institute. 2008;100:940-9.

37. Bagchi A, Papazoglu C, Wu Y, Capurso D, Brodt M, Francis D, et al. CHD5 is a tumor suppressor at human 1p36. Cell. 2007;128:459-75.

38. Maetani Y, Asano S, Mizokami A, Yamawaki Y, Sano T, Hirata M, et al. Expression of PRIP, a phosphatidylinositol 4,5-bisphosphate binding protein, attenuates $\mathrm{PI} 3 \mathrm{~K} / \mathrm{AKT}$ signaling and suppresses tumor growth in a xenograft mouse model. Biochem Biophys Res Commun. 2021;552:106-13.

39. Asano S, Taniguchi Y, Yamawaki Y, Gao J, Harada K, Takeuchi H, et al. Suppression of cell migration by phospholipase C-related catalytically inactive proteindependent modulation of PI3K signalling. Sci Rep. 2017;7:5408.

40. Chou R-H. Suppression of the invasion and migration of cancer cells by SERPINB family genes and their derived peptides. Oncol Rep. 2012;27:238-245.

41. Quan J, Adelmant G, Marto JA, Look AT, Yusufzai T. The chromatin remodeling factor CHD5 is a transcriptional repressor of WEE1. PLOS ONE. 2014;9:e108066.

42. Xie C-R, Li Z, Sun H-G, Wang F-Q, Sun Y, Zhao W-X, et al. Mutual regulation between CHD5 and EZH2 in hepatocellular carcinoma. Oncotarget. 2015;6:40940-52.

43. Edry Botzer L, Maman S, Sagi-Assif O, Meshel T, Nevo I, Yron I, et al. Hexokinase 2 is a determinant of neuroblastoma metastasis. Br J Cancer. 2016;114:759-66.

44. Egan CM, Nyman U, Skotte J, Streubel G, Turner S, O'Connell DJ, et al. CHD5 is required for neurogenesis and has a dual role in facilitating gene expression and polycomb gene repression. Dev Cell. 2013;26:223-36.
45. Dorneburg C, Fischer M, Barth TFE, Mueller-Klieser W, Hero B, Gecht J, et al. LDHA in neuroblastoma is associated with poor outcome and its depletion decreases neuroblastoma growth independent of aerobic glycolysis. Clin Cancer Res. 2018:24:5772-83.

\section{ACKNOWLEDGEMENTS}

The excellent technical assistance of Nicole Heymann, Helgard Knauss and Alix Otto is greatly appreciated. This work was supported by a grant of the Deutsche Forschungsgemeinschaft to CB (Project B1 within the GRK2254). AL was a member of the International Graduate School in Molecular Medicine, UIm, that is funded by the DFG.

\section{AUTHOR CONTRIBUTIONS}

Conception and design: CB. Development of methodology: AKL, CD. Acquisition of data: AKL. Analysis and interpretation of data (e.g., statistical analysis, biostatistics, and computational analysis): $A K L, A F, H A K, C D$, and TFEB. Writing, review, and/or revision of the paper: $C B, A K L, C D$ and $K M D$. Administrative, technical, or material support: $C B$, TFEB, and KMD. Study supervision: $C B$.

\section{FUNDING}

Open Access funding enabled and organized by Projekt DEAL.

\section{COMPETING INTERESTS}

The authors declare no competing interests.

\section{ADDITIONAL INFORMATION}

Supplementary information The online version contains supplementary material available at https://doi.org/10.1038/s41388-021-02081-0.

Correspondence and requests for materials should be addressed to Christian Beltinger.

Reprints and permission information is available at http://www.nature.com/ reprints

Publisher's note Springer Nature remains neutral with regard to jurisdictional claims in published maps and institutional affiliations.

Attribution 4.0 International License, which permits use, sharing, adaptation, distribution and reproduction in any medium or format, as long as you give appropriate credit to the original author(s) and the source, provide a link to the Creative Commons license, and indicate if changes were made. The images or other third party material in this article are included in the article's Creative Commons license, unless indicated otherwise in a credit line to the material. If material is not included in the article's Creative Commons license and your intended use is not permitted by statutory regulation or exceeds the permitted use, you will need to obtain permission directly from the copyright holder. To view a copy of this license, visit http://creativecommons. org/licenses/by/4.0/.

(c) The Author(s) 2021 